\title{
ONE-SIDED INEQUALITIES FOR THE SUCCESSIVE DERIVATIVES OF A FUNCTION
}

\author{
BY ALFRED S. CAVARETTA, JR.
}

Communicated by R. K. Miller, November 21, 1975

We fix an integer $n \geqslant 2$ and consider the class $F$ of bounded continuous functions on the positive $x$-axis satisfying

(i) $-1 \leqslant f(x) \leqslant 1$ for $x \in R^{+}$,

(ii) $f^{(n-1)}(x)$ is absolutely continuous on $R^{+}$,

(iii) $f^{(n)}(x) \leqslant 1$ a.e. on $R^{+}$.

Under these conditions on $f$, our goal is to establish best possible inequalities for the intermediate derivatives $f^{(j)}(x)$. We thus extend the work begun by Landau, further developed by Schoenberg and Cavaretta, and by Hörmander [4] [5], [2].

To settle our question for the class $F$, we need, as extremal functions, the monosplines of R. S. Johnson. A monospline of degree $n$ with $k$ knots is a function of the form

$$
M(x)=\frac{x^{n}}{n !}+\sum_{i=0}^{n-1} a_{i} x^{i}+\sum_{i=1}^{k} c_{i}\left(x-\xi_{i}\right)_{+}^{n-1}
$$

where the $a_{i}, c_{i}$, and $\xi_{i}$ are freely chosen real parameters. We note that $M^{(n-1)}(x)$ consists of $k+1$ straight line segments, each of slope 1. Considering such monosplines restricted to $[-1,1]$, Johnson [3] proves the following

Theorem. There exists a uniquely determined monospline $M_{n, k}(x)$ having precisely $n+2 k+1$ points of equioscillation on $[-1,1]$. In addition, $M_{n, k}$ has least sup norm on $[-1,1]$; i.e., $\left\|M_{n, k}\right\|_{\infty} \leqslant\|M\|_{\infty}$ with equality only if $M=M_{n, k}$.

The relevance of these functions to our class $F$ becomes apparent after we make a preliminary change of scale and origin. We consider $f(x)=a M_{n, k}(b x)$ $(a>0, b>0)$ and determine $a=a_{n, k}$ and $b=b_{n, k}$ so that $\|f\|_{\infty}=1$ on $\left[-b^{-1}\right.$, $b^{-1}$ ] and $f^{(n)}(x)=1$ except at its knots. Then define $B_{n, k}(x)=a M_{n, k}(b x-1)$ on the interval $\left[0,2 b^{-1}\right]$. In this fashion we obtain the monospline $B_{n, k}$ which on $\left[0,2 b^{-1}\right]$ is of norm 1 and has precisely $n+2 k+1$ points of equioscillation there. By elementary zero counting arguments, one readily verifies that $\operatorname{sign} B_{n, k}^{(j)}(0)=(-1)^{n+j}$.

With these preliminaries, we can now state the main theorem and its corollary.

AMS (MOS) subject classifications (1970). Primary 41 A15; Secondary 46B10. 
TheOREM. Let $1 \leqslant j \leqslant n-1$. For any $f \in F$ and any $x \geqslant 0$ we have (1) if $n+j$ is odd, then $f^{(j)}(x) \geqslant B_{n, k}^{(j)}(0)$ and no finite upper bound for $f^{(j)}(x)$ is possible; (2) if $n+j$ is even, then $f^{(j)}(x) \leqslant B_{n, k}^{(j)}(0)$ and no finite lower bound for $f^{(j)}(x)$ is possible.

Corollary. The limit $\beta_{n}^{j}=\lim _{k \rightarrow \infty} B_{n, k}^{(j)}(0)$ exists and is not equal to zero. In addition, (1) if $n+j$ is odd, $f^{(j)}(x) \geqslant \beta_{n}^{j}$ and this inequality is best possible; (2) if $n+j$ is even, $f^{(j)}(x) \leqslant \beta_{n}^{j}$ and this inequality is best possible.

Proof. Suppose $n+j$ is odd and consider statement 1 of the theorem. Since the class $F$ goes over into itself under translation to the left, it suffices to show $f^{(j)}(0) \geqslant B_{n, k}^{(j)}(0)$ for each $f \in F$. Suppose that this is not the case. So for some $f$ we have $f^{(j)}(0)<B_{n, k}^{(j)}(0)<0$. We fix $\alpha, 0<\alpha<1$, so that $B_{n, k}^{(j)}(0)-$ $\alpha f^{(j)}(0)=0$ and put $h(x)=B_{n, k}(x)-\alpha f(x)$ on the interval $\left[0,2 b^{-1}\right]$.

Since $\|\alpha f\| \leqslant \alpha<1$, equioscillation of $B_{n, k}$ implies that $h$ has $n+2 k$ distinct zeros interior to the interval $\left[0,2 b^{-1}\right]$. Hence by Rolle's theorem, $h^{(j)}$ has $n-j+2 k$ zeros in the interior of the interval. On taking account of $h(0)=$ 0 , we conclude that $h^{(j)}$ has $n-j+2 k+1$ zeros, from which it follows that $h^{(n-1)}$ must have $2 k+2$ zeros. This yields a contradiction, since from the hypothesis $f^{(n)}(x) \leqslant 1$ a. e., it follows that $h^{(n-1)}$ must consist of exactly $k+1$ increasing curves and as such can have no more than $2 k+1$ zeros. (Here, of course, we count a jump discontinuity across the $x$-axis as a simple zero.)

Concerning the lack of a finite upper bound, one need merely consider the functions $f(x)=(-1)^{n+1} M^{n}(1 / M-x)_{+}^{n}, M>0$. Clearly, $f \in F$ no matter how large $M$, and if $n+j$ is odd, $f^{(j)}(0)$ is large with $M$. The proof of statement 2 is exactly the same.

Consideration of the above shows in addition that if $n$ and $j$ are fixed with $n+j$ odd then the sequence $\left\{B_{n, k}^{(j)}(0)\right\}_{k=0}^{\infty}$ is monotonically increasing and bounded above by 0 ; hence we can set $\beta_{n}^{j}=\lim _{k \rightarrow \infty} B_{n, k}^{(j)}(0)$. Similar remarks hold for $n+j$ even. In both cases one easily verifies that $\beta_{n}^{j} \neq 0$ and that the inequalities of the corollary are valid.

Now fix an interval $[0, A]$. Using the Ascoli-Arzela theorem and extracting subsequences if necessary, we define

$$
B_{n}(x)=\lim _{k \rightarrow \infty} B_{n, k}(x) .
$$

When this is accomplished on each interval $[0, A]$, we then have a function $B_{n}(x)$ well defined on the positive axis and $B_{n}^{(j)}(0)=\beta_{n}^{j} . B_{n}(x)$ is not in $F$, although we can show that $B_{n}(x) \in C^{n-2}$. Nevertheless, by convolving $B_{n}(x)$ with, say, the Weierstrass kernel we obtain $\widetilde{B}_{n} \in F$ with values $\widetilde{B}_{n}^{(j)}(0)$ arbitrarily close to $\beta_{n}^{j}$. Thus our inequalities are best possible.

In addition to these results, one can also consider the class $F_{M}$ defined by the requirement $-M \leqslant f^{(n)}(x) \leqslant 1$ a.e. on $R^{+}$. For such a class, there are best 
possible finite upper bounds and lower bounds for each $f^{(j)}, 1 \leqslant j \leqslant n-1$. These results are handled in a fashion similar to the above. The main difference is that now there are two auxiliary functions $H_{n}(x)$ and $G_{n}(x)$ which together play the role of $B_{n}(x)$ and supply the best constants in our inequalities. As an example of these results, when $n=2$ and $f \in F_{M}$, we have $-2 \sqrt{2} \leqslant f^{\prime}(x) \leqslant$ $2 \sqrt{2 M}$. Thus the lower bound on $f^{\prime \prime}$ governs the upper bound for $f^{\prime}$. This feature persists for the higher derivatives as a dependence on the parity of $n+j$.

\section{REFERENCES}

1. A. S. Cavaretta, Jr., An elementary proof of Kolmogorov's theorem, Amer. Math. Monthly 81 (1974), 480-486. MR $49 \# 5269$.

2. L. Hörmander, $A$ new proof and a generalizaation of an inequality of Bohr, Math. Scand. 2 (1954), 33-45. MR 16, 354.

3. R. S. Johnson, On monosplines of least deviation, Trans. Amer. Math. Soc. 96 (1960), 458-477. MR 23 \#A270.

4. E. Landau, Einige Ungleichungen für zweimal differentierbare Funktionen, Proc. London Math. Soc. 13 (1913), 43-49.

5. I. J. Schoenberg, and A. S. Cavaretta, Jr., Solution of Landau's problem concerning higher derivatives on the half-line, Math. Research Center Technical Summary Report, No. 1050, University of Wisconsin, Madison, Wis., 1970.

DEPARTMENT OF MATHEMATICS, KENT STATE UNIVERSITY, KENT, OHIO 44242 